\title{
FROM THE EDITORIAL BOARD
}

With the present issue the Joint Institute for Nuclear Research initiates the series "Problems of the Physics of Elementary Particles and the Atomic Nucleus." The aim of the series is to make more widely known the most important theoretical and experimental results of investigations in the field of the physics of elementary particles and the atomic nucleus through the systematic publication of surveys on problems of topical interest written at a high scientific level. Four issues of the series are to be published each year. The individual issues will be devoted predominantly either to questions of elementary particle physics or questions of nuclear physics. The series will be referred to in the shortened form "Elementary Particles and the Atomic Nucleus (ÉChAYa) in Russian and "Particles and Nucleus" in English. The volumes and issues will be numbered.

The publication of the series "Particles and Nucleus" is needed to meet the increasing differentiation of the scientific branches of nuclear physics, a field in which thousands of highly qualified specialists are working. The publication of surveys devoted to specialized but important present-day branches of nuclear physics is made necessary to a large extent by the intensive development of these branches and the rapid accumulation of scientific information. The existing publications are not able to meet these requirements. The aim of the series is to publish such surveys as and when this becomes necessary. The series will contain review articles on subjects related to the investigations carried out at the Joint Institute for Nuclear Research. This includes theoretical and experimental problems of the physics of elementary particles, the nucleus, transuranic elements, neutron physics, problems of development of new accelerating methods and instruments, problems of automation and the mathematical processing of experimental data, and problems related to the construction of new experimental installations. The survey is also intended to acquaint the reader with new aspects of the theoretical and experimental physics of elementary particles and the atomic nucleus as they are developed in all parts of the world.

The series "Particles and Nucleus" is intended for qualified scientific researchers. Undoubtedly, it will also be very suitable for young scientific researchers, post-graduates, and students of higher courses of physics, mechanics and mathematics, and a number of engineering faculties since the surveys, written by specialists, will acquaint the reader with the problems of modern nuclear physics. It may also prove useful to other specialists not directly occupied with problems of nuclear physics in view of the considerable influence which the new experimental methods of investigation of the microscopic world exert on the technical progress of present-day society.

The publication of the series "Particles and Nucleus" will further the development of all the branches of nuclear physics that are so well represented at the Joint Institute for Nuclear Research, and have been extended to the countries that participate in the Joint Institute for Nuclear Research. 1970.

Translated from Problemy Fiziki Élementarnykh Chastits i Atomnogo Yadra, Vol. 1, No. 1, pp. 5-6,

- 1972 Consultants Bureau, a division of Plenum Publishing Corporation, 227 West 17th Street, New York, N. Y. 10011. All rights reserved. This article cannot be reproduced for any purpose whatsoever without permission of the publisher. A copy of this article is available from the publisher for $\$ 15.00$. 